PROCEEDINGS OF THE

AMERICAN MATHEMATICAL SOCIETY

Volume 140, Number 1, January 2012, Pages 207-215

S 0002-9939(2011)10903-1

Article electronically published on May 11, 2011

\title{
YOUNG MEASURES AS PROBABILITY DISTRIBUTIONS OF LOEB SPACES
}

\author{
BANG-HE LI AND TIAN-HONG LI
}

(Communicated by Richard C. Bradley)

\begin{abstract}
First we give a simple proof for a folklore result, which we call the Fundamental Theorem of Young Measures in a general framework. In the second part, we deal with the explicit representation of Young measures on Euclidean spaces. Young measure is an abstract concept in the sense that when it is described, it needs all continuous functions $\phi(y)$ and all $L^{1}$ functions $f(x)$ in the realm of standard analysis. However, we found that in the framework of nonstandard analysis, Young measures at almost all points are proved to be probability distributions for some random variables on some Loeb spaces defined in the monads of those points. This means that we can describe this Young measure without using $f(x)$ and $\phi(y)$. This also leads to the concrete computation of Young measures.
\end{abstract}

\section{Fundamental theorem of Young measures}

Young measures were introduced by Young as generalized curves and surfaces in [25, 26, 27] and followed by McShane in [15, 16] in the 1930s and 1940s in studying variational problems. Some analogous approaches were presented by Warga in 22, 23, 24] in the 1960s. In [24], $X$ and $Y$ are assumed to be metric and compact spaces such that there is a positive complete measure $d x$ on $X$. Let $\mathcal{B}$ be the space of functions $\phi$ on $X \times Y$, continuous on $Y$ for every $x \in X$, measurable on $X$ for every $y \in Y$ and with $\sup _{y \in Y}|\phi(x, y)|$ integrable on $X$. Then a Young measure is regarded as an element $\nu$ in the dual space of the Banach space $\mathcal{B}$ with norm $|\phi|=\int_{X} \sup _{y \in Y}|\phi(x, y)| d x$ with the properties $\langle\nu, \phi\rangle \geq 0$ for every nonnegative $\phi$ in $\mathcal{B}$ and $\langle\nu, \bar{\phi}\rangle=\int_{E} d x$ if $\bar{\phi}(x, y)=1$ on $E \times Y$ and $\bar{\phi}(x, y)=0$ on $(X-E) \times Y$ for

Received by the editors June 14, 2010 and, in revised form, November 3, 2010.

2010 Mathematics Subject Classification. Primary 28E05, 28A20, 26E35; Secondary 03H10, $49 \mathrm{~J} 45$.

Key words and phrases. Young measure, representation, nonstandard analysis, weak-star convergence, Loeb measure.

The first author is partially supported by the National Natural Science Foundation of China (NSFC) under Grant No. 10771206 and partially by 973 project (2004CB318000) of the People's Republic of China.

The second author is supported by the Youth Foundation by Chinese NSF 10701073 and Chinese NSF 10931007, and is partially supported by the National Center for Theoretical Sciences and the National Tsing Hua University in Taiwan. Both authors thank the referee for many helpful comments. 
every measurable $E \subset X . \nu$ can also be regarded as a measurable function from $X$ to the space of regular Borel probability measures on $Y$ with weak topology, such that $\langle\nu, \phi\rangle=\int_{X} d x \int_{Y} \phi(x, y) d \nu_{x}(y)$.

Denote the set of Young measures by $M(d x, Y)$. Let $\rho$ be a measurable function from $X$ to $Y$, viewed as an element of $M(d x, Y)$ by mapping $\phi$ into the number $\int_{X} \phi(x, \rho(x)) d x$. Denote by $F(d x, Y)$ the set of all such $\rho$. Assuming that the measure $d x$ is finite, regular, complete and nonatomic, Warga proved in 24] that with respect to the weak star topology, $M(d x, Y)$ is sequentially compact and $F(d x, Y)$ is dense in $M(d x, Y)$.

Berliocchi and Lasry in [5 (1973) studied the case of $X$ and $Y$ being locally compact, separable and metrizable topological spaces such that $d x$ is a positive complete Radon measure on $X$. Then a Young measure, also called a parameterized measure in [5], is a Radon measure on $X \times Y$ with $d x$ as its projection on $X$. Equivalently, a Young measure is a measurable function from $X$ to the space of Radon probability measures on $Y$ with the weak topology. Here, the weak topology on the space of measures on $Y$ is given by the family of semi-norms relevant to $C_{c}(Y)$, the space of continuous functions on $Y$ with compact support. Denote by $M(d x, y)$ the space of Young measures with the weak topology given by $C_{c}(X \times Y)$, and $F(d x, Y)$ is the subset of $M(d x, Y)$ given by all measurable functions $X \longrightarrow Y$. It is proved in 5 that if $Y$ is compact, then $M(d x, y)$ is a metrizable compact space, and if $d x$ is nonatomic, then $F(d x, Y)$ is dense in $M(d x, y)$.

The last section of [5] is devoted to comments on the generalization of their results to the case of $X$ being only a set with a $\sigma$-algebra, and $d x$ is a complete $\sigma$-finite measure without a proof, indicated to them by Castaing.

For a quite long period since then, several authors studying Young measures seem still to have been unaware of this generalization, as Balder showed in the review (MR 1036070 (91c: 49021)) of the paper of Ball [4] in 1989. Balder in a series of papers (cf. [3]) studied Young measures in this general setting; also Castaing et al. published a book [7] in 2004.

Here we give a simple proof for this generalization and call it the Fundamental Theorem of Young Measures, so that this section of our paper can be regarded as a self-contained introduction to the general approach to Young measures. Since the one-point compactification of locally compact, separable and metrizable spaces is compact and metrizable, we may assume that $Y$ is a compact metric space without loss of generality.

Fundamental Theorem of Young Measures. Let $X$ be any set with a $\sigma$-finite complete measure $d x, Y$ a compact metric space. Then

1) The space of Young measures $M(d x, Y)$ is compact with the weak star topology given by $L^{1}\left(d x, C_{Y}\right)$. Furthermore, if $L^{1}(d x)$ is separable, then $M(d x, Y)$ is metrizable.

2) For every $\nu \in M(d x, Y)$, for almost all $x, \nu_{x}$ is a probability measure. For any $\phi \in C_{Y}$, the map from $M(d x, Y)$ to $L^{\infty}(d x)$ given by $\nu \longrightarrow \nu_{\phi}$ with $\nu_{\phi}(x)=$ $\int_{Y} \phi(y) d \nu_{x}(y)$ is continuous with respect to the weak star topology of $L^{\infty}(d x)$ as the dual of $L^{1}(d x)$. 
3) If $d x$ is nonatomic, then the set $F(d x, Y)$ given by measurable functions: $X \longrightarrow Y$ is dense in $M(d x, Y)$ with respect to the weak star topology.

Proof. Let $\mathcal{B}$ be the space of Carathéodory functions $\phi(x, y)$ on $X \times Y$; that is, $\phi(x, \cdot)$ is continuous for almost all $x$, and $\phi(\cdot, y)$ is measurable for every $y$, such that $\sup _{y}|\phi(x, y)|=|\phi(x, \cdot)|_{C_{Y}}$ is integrable, where $|\cdot|_{C_{Y}}$ stands for norm of the Banach space $C_{Y}$ formed by all continuous functions on $Y$. Then by Theorem 11, p. 149, and Theorem 22, p. 117, in [11, we have that $\mathcal{B}=L^{1}\left(d x, C_{Y}\right)$, the Banach space of integrable functions $f$ from $X$ to $C_{Y}$ with norm $|f|_{\mathcal{B}}=\int_{X}|f(x)|_{C_{Y}} d x$. Denote by $L^{1}(d x)$ the space of integrable functions. Then the linear space generated by $f(x) \phi(y), f \in L^{1}(d x), \phi \in C_{Y}$, is dense in $\mathcal{B}$ according to [17, $\S 6.5$, p. 96.

Let $\mathcal{B}^{*}$ denote the dual space of $\mathcal{B}$. For any $\nu \in \mathcal{B}^{*}, f \in L^{1}(d x), \phi \in C_{Y}$, we have $|\langle\nu, f \phi\rangle| \leq|\nu||f||\phi|$, where $|\cdot|$ is understood in an obvious way. Then for fixed $\nu$ and $\phi,\langle\nu, f \phi\rangle$ defines a bounded linear functional on $L^{1}(d x)$. Thus, there is an element in $L^{\infty}(d x)$ denoted by $\nu_{\phi}$ such that $\langle\nu, f \phi\rangle=\int_{X} f(x) \nu_{\phi}(x) d x$ by Theorem 7.4-B, p. 383, in 20. Denote by $\bar{L}^{\infty}(d x)$ the space of bounded measurable functions $b$ on $X$ with norm $|b|_{\bar{L}^{\infty}}=\sup _{x}|b(x)|$. Since $C_{Y}$ is separable, and for a fixed $\nu$, $\phi \longrightarrow \nu_{\phi}$ is bounded linear from $C_{Y}$ to $L^{\infty}(d x),\left\{\nu_{\phi} \mid \phi \in C_{Y}\right\}$ is a separable subset of $L^{\infty}(d x)$. By the Dunford-Pitts lemma (cf. [18, Exposé no. 4, pp. 1-2), there is a linear functional: $\left\{\nu_{\phi} \mid \phi \in C_{Y}\right\} \longrightarrow \bar{L}^{\infty}$ preserving the norms, so we may choose a representation $\bar{\nu}_{\phi}$ of $\nu_{\phi}$ for any $\phi \in C_{Y}$ such that $\left|\bar{\nu}_{\phi}\right|_{\bar{L}^{\infty}}=\left|\nu_{\phi}\right|_{L^{\infty}}$. Therefore $\phi \longrightarrow \bar{\nu}_{\phi}$ is bounded linear from $C_{Y} \longrightarrow \bar{L}^{\infty}(d x)$. Now, for any $x \in X, \phi \longrightarrow \bar{\nu}_{\phi}(x)$ is a bounded linear functional on $C_{Y}$, hence corresponds to a Radon measure $\nu_{x}$ on $Y$ such that $\bar{\nu}_{\phi}(x)=\int_{Y} \phi(y) d \nu_{x}(y)$ by the Riesz representation theorem.

Let $M(d x, Y)$ be the space of Young measures $\nu$. By the definition of Young measures and the separability of $C_{Y}, \nu_{x}$ can be regarded as a probability measure for almost all $x$. Thus we have proved 2). It is easy to prove that $M(d x, Y)$ is a closed subset of the unit ball of $\mathcal{B}^{*}$ with the weak star topology, hence a compact space by the Banach-Alaoglu theorem. Letting $F(d x, Y)$ be the subset of $M(d x, Y)$ given by all measurable functions: $X \longrightarrow Y$, the density result is deduced from the Lyapunov convexity theorem for nonatomic finite measures. The proof is thus complete.

Remark 1.1. For $X$ being a locally compact, separable and metrizable space, the equivalence of $M(d x, Y)$ viewed as subsets of the dual of $\mathcal{B}$ and $C_{c}(X \times Y)$ can be proved by the following fact: the set consisting of $\phi \varphi, \phi \in C_{c}(X), \varphi \in C_{c}(Y)=C_{Y}$, is dense both in $\mathcal{B}$ and $C_{c}(X \times Y)$. The density in $\mathcal{B}$ follows from the density of $C_{c}(X)$ in $L^{1}(d x)$. To prove the density in $C_{c}(X \times Y)$, one might appeal to the partitions of unity on $X$.

Near the end of the 1970s, in the study of the compensated compactness method to conservation laws, Tartar reproved the fundamental theorem of the Young measure in the following special case: $X$ is a bounded open set in $\mathbf{R}^{m}$ with $d x$ the Lebesgue measure, and $Y$ is the closure of a bounded open set in $\mathbf{R}^{n}$ (cf. 21], [10] and [6]). By applying the compensated compactness method to more general cases, P. Lin 13 re-proved 1) and 2) of the fundamental theorem in a slightly weaker form for the following case: $X$ is a measurable subset of $\mathbf{R}^{m}$, and $Y=\mathbf{R}^{n} \cup\{\infty\}=S^{n}$. 
Remark 1.2. There is a gap in the proof of Lin's Theorem 2.1 concerning Young measures. That is, on p. 382 in [13], it says that "we suppose, without loss of generality, that every $x \in \Omega$ is a Lebesgue point of each $\alpha(f)$." Actually $\alpha(f)$ as an element of $L^{\infty}$ is defined as an equivalence class of $\bar{L}^{\infty}$, while for a point that is a Lebesgue point, it depends on choosing a representative $\bar{\alpha}(f)$ of $\alpha(f)$ in $\bar{L}^{\infty}$. Therefore, a use of the Dunford-Pitts Lemma is needed to guarantee almost all $x \in \Omega$ being Lebesgue points for all $\bar{\alpha}(f) \in \bar{L}^{\infty}$ with $f \in C_{c}\left(\mathbf{R}^{n}\right)$.

\section{Representation of Young Measures}

Hereafter, we turn to represent Young measures by internal measurable functions in the framework of nonstandard analysis, which gives a local picture for Young measures. We refer the reader to [19] for an introduction to nonstandard analysis.

To guarantee the nonstandard model having the properties we need, we simply assume that the model here is multi-saturated.

Notice that the pioneer work on representing Young measures by internal hyperfinite functions is due to Cutland [8]. See also Arkeryd [2] for a relevant work on Loeb solutions for Boltzmann equations. Here, we represent Young measure in a different way in the case of $X$ being a measurable subset of $\mathbf{R}^{m}$ with $d x$ the Lebesgue measure, and $Y$ a compact subset of $\mathbf{S}^{n}=\mathbf{R}^{n} \cup\{\infty\}$.

In Theorem 2.1 below, the following will be obtained: for any internal measurable function $u:{ }^{*} X \longrightarrow{ }^{*} Y, u \in{ }^{*} F(d x, Y)$, it follows from the compactness of $M(d x, Y)$ and the nonstandard criterion of compactness due to Robinson that there is a $\nu \in M(d x, Y)$ such that $u$ is in the monad of $\nu$ in ${ }^{*} M(d x, Y)$.

There we will also give a formula for $\nu_{x}$ directly by $u$ and Loeb measures. For any $x \in \mathbf{R}^{m}$, and any internal measurable subset $A$ of the monad of $x$ with positive measure, let $d_{L} x$ be the Loeb measures of $A$ induced from the internal Lebesgue measure on $A$ and normalized to a probability measure (see 14] or 9]). Using ${ }^{\circ} a$ or $s t(a)$ to represent the standard part of a nonstandard object $a$, then $x \longrightarrow{ }^{o} u(x)$ defines a Loeb measurable function $A \longrightarrow Y$ (see [1] or [9]). We will prove that for certain $A, \nu_{x}$ is just the probability distribution of the function ${ }^{\circ} u(x)$ with respect to the Loeb probability measure on $A$. More precisely, it is

Theorem 2.1. Let $u:{ }^{*} \mathbf{R}^{m} \longrightarrow{ }^{*} Y$ be an internal Lebesgue measurable function with $Y$ a compact subset of $\mathbf{S}^{n}=\mathbf{R}^{n} \cup\{\infty\}$. Then

(1) there is a Young measure $\nu$ such that

$$
\int_{\mathbf{R}^{m}} f(x) d x \int_{Y} \phi(y) d \nu_{x}(y)=s t\left(\int_{* \mathbf{R}^{m}}{ }^{*} \phi(u(x))^{*} f(x) d x\right)
$$

for any $f \in L^{1}(d x)$ and $\phi \in C_{Y}$.

(2) For almost all points $x_{0}$ in $\mathbf{R}^{m}$ and any bounded measurable set $B \subset \mathbf{R}^{m}$ with positive Lebesgue measure, there exists a positive infinitesimal $\rho_{0}$ such that if $\rho \geq \rho_{0}$ is an infinitesimal, then $\nu_{x_{0}}$ is the probability distribution of ${ }^{o} u(x)$ defined on $x_{0}+\rho^{*} B=\left\{x \in{ }^{*} \mathbf{R}^{m} \mid x=x_{0}+\rho b, b \in{ }^{*} B\right\}$ with the natural Loeb probability measure, and for any $\phi \in C_{Y}$,

$$
\nu_{\phi}\left(x_{0}\right)=\int_{Y} \phi(y) d \nu_{x_{0}}(y)=\int_{\rho^{*} B} \phi\left({ }^{o} u\left(x_{0}+b\right)\right)^{o}\left(\frac{d b}{\rho^{m}|B|}\right),
$$

where $|B|$ is the Lebesgue measure of $B$.

(3) Conversely, every Young measure is obtained by some such $u$. 
Remark 2.2. If $u(x) \in{ }^{*} \mathbf{S}^{n} \backslash O\left(\mathbf{R}^{n}\right)$, then ${ }^{o} u(x)=\infty$.

If $u^{j}(x): \mathbf{R}^{m} \rightarrow Y$ are measurable functions, then by the Fundamental Theorem of Young Measures 1), there exists a subsequence (still denoted by) $u^{j}(x)$ and a family of probability measures $\nu_{x} \in \operatorname{Prob}\left(\mathbf{R}^{m}\right), x \in \mathbf{R}^{m}$, with $\operatorname{supp} \nu_{x} \subset Y$, such that for any continuous function $\phi: Y \rightarrow \mathbf{R}$ and any function $f(x) \in L^{1}(d x)$, it follows that

$$
\lim _{j \rightarrow \infty} \int_{\mathbf{R}^{m}} f(x) \phi\left(u^{j}(x)\right) d x=\int_{\mathbf{R}^{m}}\left(f(x) \int_{Y} \phi(y) d \nu_{x}(y)\right) d x .
$$

We say that the Young measure $\nu$ is the limit of this sequence of measurable functions $\left\{u^{j}\right\}_{j=1}^{\infty}$ or that this Young measure $\nu$ corresponds to this sequence $\left\{u^{j}\right\}_{j=1}^{\infty}$ if equation (2.2) holds.

A direct and simple application of Theorem 2.1 to the function sequences is the following:

Corollary 2.3. Suppose $Y$ is a compact subset of $\mathbf{S}^{n}=\mathbf{R}^{n} \cup\{\infty\}$. Let $\left\{u^{j}\right\}_{j=1}^{\infty}$ : $\mathbf{R}^{m} \rightarrow Y$ be a sequence of measurable functions, and let a Young measure $\nu$ be the limit of $\left\{u^{j}\right\}_{j=1}^{\infty}$. Then for any $\omega \in^{*} \mathbf{N} \backslash \mathbf{N}$, we have

$$
\int_{\mathbf{R}^{m}} f(x) d x \int_{Y} \phi(y) d \nu_{x}(y)=\operatorname{st}\left(\int_{{ }^{*} \mathbf{R}^{m}} * \phi\left(u^{\omega}(x)\right)^{*} f(x) d x\right)
$$

for any $f \in L^{1}(d x)$ and $\phi \in C_{Y}$. For almost all points $x_{0}$ in $\mathbf{R}^{m}$ and any bounded measurable set $B \subset \mathbf{R}^{m}$ with positive Lebesgue measure, there exists a positive infinitesimal $\rho_{0}$ such that if $\rho \geq \rho_{0}$ is an infinitesimal, then the Young measure $\nu_{x_{0}}$ is the probability distribution of ${ }^{o} u^{\omega}(x)$ defined on $x_{0}+\rho^{*} B=\left\{x \in{ }^{*} \mathbf{R}^{m} \mid x=\right.$ $\left.x_{0}+\rho b, b \in{ }^{*} B\right\}$ with the natural Loeb probability measure, and for any $\phi \in C_{Y}$,

$$
\text { weak }{ }^{*} \lim \phi\left(u^{j}\right)\left(x_{0}\right)=\int_{Y} \phi(y) d \nu_{x_{0}}(y)=\int_{\rho^{*} B} \phi\left({ }^{o} u^{\omega}\left(x_{0}+b\right)\right)^{o}\left(\frac{d b}{\rho^{m}|B|}\right),
$$

where $|B|$ is the Lebesgue measure of $B$.

We can apply this corollary to compute Young measures for some simple examples. Firstly, let $I(x)$ be a periodic function with period 1 such that $I(x)=1-|4 x|$ when $x \in[-1 / 2,1 / 2]$. Then for any $x$, the corresponding Young measure $\nu_{x}$ for $\{I(j x)\}_{j=1}^{\infty}$ is the uniform distribution on $[-1,1]$. Next, for any $x$, the density of the Young measures $\nu_{x}$ for $\{\sin (j x)\}_{j=1}^{\infty}$ is $\frac{1}{\pi}(\arcsin y)^{\prime}=\frac{1}{\pi} \frac{1}{\sqrt{1-y^{2}}}$ supported on $[-1,1]$. More generally, let $f(x)$ be an $L_{l o c}^{1}$ function. Then for any Lebesgue point $x$ of $f$, the corresponding Young measure $\nu_{x}$ for $\{f(x) I(j x)\}_{j=1}^{\infty}$ is the uniform distribution on $[-|f(x)|,|f(x)|]$. Similarly, for any Lebesgue point $x$ of $f$, if $f(x) \neq 0$, then the density of the Young measures $\nu_{x}$ for $\{f(x) \sin (j x)\}_{j=1}^{\infty}$ is $\frac{1}{\pi} \frac{1}{\sqrt{|f(x)|^{2}-y^{2}}}$ supported on $[-|f(x)|,|f(x)|]$. If $f(x)=0$, then the Young measure $\nu_{0}$ is the Dirac measure at 0 . Finally, consider $X=[0,1]$ with Lebesgue measure, and

$$
u^{j}(x)= \begin{cases}j, & \text { if } x \in[0,1 / j] \\ 0, & \text { otherwise. }\end{cases}
$$

Then $\nu_{x}=\delta_{0}$ for each $x \in[0,1]$.

Since we have described Young measures in the nonstandard space in a rather beautiful way, we hope to describe them in the standard space in the same spirit. However, we have only the following: 
Corollary 2.4. Suppose $Y$ is a compact subset of $\mathbf{S}^{n}=\mathbf{R}^{n} \cup\{\infty\}$. Let $\left\{u^{j}\right\}_{j=1}^{\infty}$ : $\Omega \rightarrow Y$ be a sequence of measurable functions converging to a Young measure $\nu$. Then for almost all points $x_{0}$ in $\Omega$ and any bounded measurable set $B \subset \mathbf{R}^{m}$ with positive Lebesgue measure, $\forall \epsilon>0, \forall \delta>0, \forall \phi \in C_{Y}, \exists j_{0} \in \mathbf{N}, \forall j \in \mathbf{N}$ and $j \geq j_{0}$, $\exists \delta_{0}, \delta_{1}$ such that $\delta_{0}<\delta_{1} \leq \delta$, for any $\tilde{\delta} \in\left[\delta_{0}, \delta_{1}\right]$, we have

$$
\left|\int_{Y} \phi(y) d \nu_{x_{0}}(y)-\frac{1}{|\tilde{\delta} B|} \int_{\tilde{\delta} B} \phi\left(u^{j}\left(x_{0}+b\right)\right) d b\right|<\epsilon .
$$

Remark 2.5. Notice that in this standard version, the estimates depend on $\phi$. For different $\phi$, we may have different sets $x_{0}+\tilde{\delta} B$ with $\tilde{\delta}$ depending on $\phi$. So we see that the standard version is not as beautiful as the nonstandard one.

\section{Proof of Theorem 2.1}

By a similar argument as in the proof of the Fundamental Theorem of Young Measures, the set $M(d x, Y)$ of Young measures $\nu$ can be identified with the bounded linear operators: $C_{Y} \longrightarrow L^{\infty}(d x)$ between the two Banach spaces given by $\phi \longrightarrow \nu_{\phi}$ such that $\phi \geq 0$ implies $\nu_{\phi} \geq 0$ and $\phi \equiv 1$ implies $\nu_{\phi} \equiv 1$. If $h: X \longrightarrow Y$ is a measurable function, the corresponding $\nu_{\phi}$ is given by $\nu_{\phi}(x)=\phi(h(x))$.

Now let $u$ be an internal measurable function: ${ }^{*} \mathbf{R}^{m} \longrightarrow{ }^{*} Y$. Then ${ }^{*} \phi(u(x))$ is an element in ${ }^{*} L^{\infty}(d x)$ with finite norm. We will need the following nonstandard criterion of compactness of Robinson (see [8] or [19]):

Let $Z$ be a Hausdorff space. $Z$ is compact if and only if any element $a$ in ${ }^{*} Z$ is a near standard point, i.e., $\exists$ a unique element $x \in Z$ such that $a \approx x$.

The compactness of the unit ball of $L^{\infty}(d x)$ with the weak star topology (BanachAlaoglu theorem), and the nonstandard criterion of compactness of Robinson imply that there is a unique $\nu_{\phi} \in L^{\infty}(d x)$ such that

$$
\int_{\mathbf{R}^{m}} \nu_{\phi}(x) f(x) d x \approx \int_{* \mathbf{R}^{m}} * \phi(u(x))^{*} f(x) d x
$$

for any $f \in L^{1}(d x)$. Therefore there is a bounded linear operator given by $\phi \longrightarrow \nu_{\phi}$ such that $\phi \geq 0$ implies $\nu_{\phi} \geq 0$ and $\phi \equiv 1$ implies $\nu_{\phi} \equiv 1$. So there is a Young measure $\nu$. By the Fundamental Theorem of Young Measures, $\nu_{x}$ is a probability measure for almost all $x$ with

$$
\nu_{\phi}(x)=\int_{Y} \phi(y) d \nu_{x}(y), \text { a.e. } x \text {. }
$$

Thus part 1 is proved.

Since $C_{Y}$ is separable, take a countable dense subset $\left\{\phi_{k}\right\}$ of $C_{Y}$. Then there is a subset $E$ of $\mathbf{R}^{m}$ such that $\mathbf{R}^{m}-E$ has measure zero and any $x_{0} \in E$ is a Lebesgue point for any $\nu_{\phi_{k}}$. We claim that for any $\phi \in C_{Y}, x_{0}$ is also a Lebesgue point of $\nu_{\phi}$; i.e.,

$$
\lim _{h \longrightarrow 0} \frac{1}{h^{m}} \int_{Q_{h}}\left|\nu_{\phi}\left(x+x_{0}\right)-\nu_{\phi}\left(x_{0}\right)\right| d x=0,
$$

where $Q_{h}=\left\{x \in \mathbf{R}^{m}|\quad| x_{i} \mid<h / 2, i=1,2, \ldots, m\right\}$. For any $\varepsilon>0$, take a $\phi_{k}$ such that $\left|\phi(y)-\phi_{k}(y)\right|<\varepsilon$ for any $y \in Y$. Then $\left|\nu_{\phi}(x)-\nu_{\phi_{k}}(x)\right|<\varepsilon$ for any $x \in X$. Now

$$
\begin{aligned}
\left|\nu_{\phi}\left(x+x_{0}\right)-\nu_{\phi}\left(x_{0}\right)\right| \leq \quad & \left|\nu_{\phi}\left(x+x_{0}\right)-\nu_{\phi_{k}}\left(x+x_{0}\right)\right|+\left|\nu_{\phi}\left(x_{0}\right)-\nu_{\phi_{k}}\left(x_{0}\right)\right| \\
& +\left|\nu_{\phi_{k}}\left(x+x_{0}\right)-\nu_{\phi_{k}}\left(x_{0}\right)\right| .
\end{aligned}
$$


Hence

$$
\varlimsup_{h \longrightarrow 0} \frac{1}{h^{m}} \int_{Q_{h}}\left|\nu_{\phi}\left(x+x_{0}\right)-\nu_{\phi}\left(x_{0}\right)\right| d x<2 \varepsilon .
$$

The claim is proved.

We need the following definition and lemma (Theorem 2 in [12]):

Definition 3.1. Let $g$ be an internal Lebesgue integrable function on ${ }^{*} \mathbf{R}^{m}$. We say that $g$ is a nonstandard representation of a distribution $\tilde{g}$ on $\mathbf{R}^{m}$ if for any $\phi \in \mathscr{D}\left(\mathbf{R}^{m}\right)$,

$$
\int_{* \mathbf{R}^{m}} g^{*} \phi d x \approx\langle\tilde{g}, \phi\rangle .
$$

Lemma 3.2. Let $\tilde{g} \in L_{\text {loc }}^{1}\left(\mathbf{R}^{m}\right), x_{0}$ be a Lebesgue point of $\tilde{g}$, and $B$ be any bounded standard measurable set in $\mathbf{R}^{m}$ with Lebesgue measure $|B|>0$, where $g$ is a nonstandard representation of $\tilde{g}$. Then there is an infinitesimal $\rho_{0}$ such that for any infinitesimal $\rho \geq \rho_{0}$,

$$
\frac{1}{\rho^{m}|B|} \int_{\rho^{*} B} g\left(x_{0}+b\right) d b \approx \tilde{g}\left(x_{0}\right) .
$$

From (3.1), we can see that ${ }^{*} \phi(u(x))$ is a nonstandard representation of $\nu_{\phi}(x)$. By Lemma 3.2, for any $x_{0} \in E$ and bounded measurable set $B$ in $\mathbf{R}^{m}$ with positive Lebesgue measure, there is a positive infinitesimal $\rho_{0, k}$ such that for any infinitesimal $\rho \geq \rho_{0, k}$,

$$
\nu_{\phi_{k}}\left(x_{0}\right) \approx \frac{1}{\rho^{m}|B|} \int_{\rho^{*} B}{ }^{*} \phi_{k}\left(u\left(x_{0}+b\right)\right) d b .
$$

By Robinson's lemma, there is an infinitesimal $\rho_{0} \geq \rho_{0, k}$ for any $k$. Then it is easy to see that for any $\phi \in C_{Y}$ and infinitesimal $\rho \geq \rho_{0}$,

Therefore

$$
\nu_{\phi}\left(x_{0}\right) \approx \frac{1}{\rho^{m}|B|} \int_{\rho^{*} B}^{*} \phi\left(u\left(x_{0}+b\right)\right) d b .
$$

$$
\nu_{\phi}\left(x_{0}\right)=\int_{\rho^{*} B} \phi\left({ }^{o} u\left(x_{0}+b\right)\right) d_{L} b=\int_{Y} \phi(y) d \nu_{x_{0}}(y),
$$

where $d_{L} b={ }^{o}\left(\frac{d b}{\rho^{m}|B|}\right)$. This shows that $\nu_{x_{0}}$ is the probability distribution of the function ${ }^{\circ} u(x)$ defined on the Loeb probability space $x_{0}+\rho^{*} B$, and the proof of part 2 is complete. Part 3 is obtained by the density of $F(d x, Y)$ in $M(d x, Y)$.

Remark 3.3. The referee points out that the proof of Theorem 2.1 could be modified to yield a generalization with the finite-dimensional Euclidean space $\mathbf{R}^{m}$ substituted by a hyperfinite version (taking $m \in{ }^{*} \mathbf{N} \backslash \mathbf{N}$ together with the appropriate Loeb measure) and $\omega_{1}$-saturation is sufficient for applying Robinson's characterization in the proof of Theorem 2.1 as Y is a subset of a finite-dimensional Euclidean space.

Proof of Corollary 2.3; $\nu$ is the Young measure corresponding to $\left\{u^{j}(x)\right\}_{j=1}^{\infty}$; that is, for any $f \in L^{1}(d x)$ and $\phi \in C_{Y}$,

$$
\int_{\mathbf{R}^{m}} f(x) d x \int_{Y} \phi(y) d \nu_{x}(y)=\lim _{j \rightarrow \infty} \int \phi\left(u^{j}(x)\right) f(x) d x .
$$

Then for any $\omega \in^{*} \mathbf{N} \backslash \mathbf{N}$, we have

$$
\int_{\mathbf{R}^{m}} f(x) d x \int_{Y} \phi(y) d \nu_{x}(y) \approx \int_{{ }^{*} \mathbf{R}^{m}}{ }^{*} \phi\left(u^{\omega}(x)\right)^{*} f(x) d x .
$$

We can apply Theorem 2.1 where the internal function $u=u^{\omega}$. 
Proof of Corollary 2.4; From Corollary 2.3, we know that for almost all $x_{0} \in \mathbf{R}^{m}$, $\forall \phi \in C_{Y}, \forall \epsilon \in \mathbf{R}_{+}, \forall \delta \in \mathbf{R}_{+}$, we have the following sentence:

$\exists \omega_{0} \in{ }^{*} \mathbf{N}, \forall \omega \in{ }^{*} \mathbf{N}$ and $\omega \geq \omega_{0}, \exists \rho_{0}, \rho_{1} \in{ }^{*} \mathbf{R}$ such that $\rho_{0}<\rho_{1}<\delta$, and $\forall \rho \in{ }^{*} \mathbf{R}, \rho \in\left[\rho_{0}, \rho_{1}\right]$ implies

$$
\left|\int_{Y} \phi(y) d \nu_{x_{0}}(y)-\frac{1}{\rho^{m}|B|} \int_{\rho^{*} B} * \phi\left(u^{\omega}\left(x_{0}+b\right)\right) d b\right|<\epsilon .
$$

Now by using the Transfer Principle in nonstandard analysis, we obtain

$\exists j_{0} \in \mathbf{N}, \forall j \in \mathbf{N}$ and $j \geq j_{0}, \exists \delta_{0}, \delta_{1} \in \mathbf{R}$ such that $\delta_{0}<\delta_{1}<\delta$, and $\forall \tilde{\delta} \in \mathbf{R}$, $\tilde{\delta} \in\left[\delta_{0}, \delta_{1}\right]$ implies

$$
\left|\int_{Y} \phi(y) d \nu_{x_{0}}(y)-\frac{1}{\tilde{\delta}^{m}|B|} \int_{\tilde{\delta} B} \phi\left(u^{j}\left(x_{0}+b\right)\right) d b\right|<\epsilon .
$$

The proof is complete.

\section{REFERENCES}

1. R. M. Anderson, Star-finite representations of measure space, Trans. Amer. Math. Soc. 271 (1982) 667-687. MR654856 (83m:03077)

2. L. Arkeryd, Loeb solutions of the Boltzmann equation, Arch. Ration. Mech. Anal. 86 (1984) 85-97. MR748925 (85m:76047)

3. E. J. Balder, A general approach to lower semicontinuity and lower closure in optimal control theory, SIAM J. Control Optim. 22 (1984) 570-598. MR747970 (85k:49018)

4. J. M. Ball, A version of the fundamental theorem for Young measures, Proc. Conf. on Partial Differential Equations and Continuum Models of Phase Transitions (Nice, 1988) (D. Serre, ed.), Springer, 1989. MR1036070 (91c:49021)

5. H. Berliocchi, J.M. Lasry, Intégrandes normales et mesures paramétrées en calcul des variations, Bull. Soc. Math. France 101 (1973) 129-184. MR0344980 (49:9719)

6. G.-Q. Chen, Compactness Methods and Nonlinear Hyperbolic Conservation Laws, AMS/IP Studies in Advanced Mathematics, Vol. 15, Amer. Math. Soc., 2000, 33-75. MR1767623 (2001f:35249)

7. C. Castaing, P. Raynaud de Fitte, M. Valadier, Young measures on topological spaces. With applications in control theory and probability theory. Mathematics and Its Applications, 571. Kluwer Academic Publishers, Dordrecht, 2004. MR.2102261 (2005k:28001)

8. N. J. Cutland, Internal controls and relaxed controls, J. London Math. Soc. (2) 27 (1983) 130-140. MR686511 (84i:49019)

9. N. J. Cutland, Loeb Measures in Practice: Recent Advances, Lecture Notes in Math., 1751, Springer-Verlag, 2000. MR1810844 (2002k:28018)

10. B. Dacorogna, Weak Continuity and Weak Lower Semi-continuity of Non-linear Functionals, Lecture Notes in Math., 922, Springer-Verlag, 1982. MR658130(84f:49020)

11. N. Dunford, J. Schwartz, Linear Operators, Part I. General Theory, Interscience, New York, 1958. MR0117523(22:8302)

12. B.-H. Li, On the moiré problem from distributional point of view, J. Sys. Sci. \& Math. Sci., 6 (4) (1986) 263-268. MR877260 (88i:03107)

13. P. Lin, Young measures and an application of compensated compactness to one-dimensional nonlinear elastodynamics, Trans. Amer. Math. Soc. 329 (1992) 377-413. MR 1049615 (92e:35106)

14. P. A. Loeb, Conversion from nonstandard to standard measure spaces and applications in probability theory, Trans. Amer. Math. Soc. 211 (1975) 113-122. MR0390154(52:10980)

15. E. McShane, Generalized curves, Duke Math. J. 6 (1940), 513-536. MR0002469 (2:59d)

16. E. McShane, Necessary conditions in generalized-curve problems of the calculus of variations, Duke Math. J. 7 (1940) 1-27. MR0003478 (2:226a)

17. H. H. Schaefer, Topological Vector Spaces, Macmillan, New York, 1966. MR0193469 (33:1689)

18. L. Schwartz, Produits tensoriels topologiques d'espaces vectoriels topologiques, Espaces vectoriels topologiques nucléaires. Applications, Séminaire 1953/1954, Faculté des Sciences de Paris, 1954. 
19. K. D. Stroyan, W. A. J. Luxemburg, Introduction to the Theory of Infinitesimals, Academic Press, New York, 1976. MR0491163 (58:10429)

20. A. E. Taylor, Introduction to Functional Analysis, John Wiley \& Sons, Inc., 1963. MR.0098966 (20:5411)

21. L. Tartar, Compensated compactness and applications to partial differential equations, HeriotWatt Symposium, Vol. 4, Pitman, 1979. MR0584398 (81m:35014)

22. J. Warga, Relaxed variational problems, J. Math. Anal. Appl. 4 (1962) 111-128. MR0142020 $(25: 5415 \mathrm{a})$

23. J. Warga, Necessary conditions for minimum in relaxed variational problems, J. Math. Anal. Appl. 4 (1962) 129-145. MR0142021 (25:5415b)

24. J. Warga, Functions of relaxed controls, SIAM J. Control 5 (1967) 628-641. MR0226474 $(37: 2064 \mathrm{a})$

25. L. C. Young, Generalized curves and the existence of an attained absolute minimum in the calculus of variations, C. R. Soc. Sci. Lett. Varsovie, Classe III, 30 (1937) 212-234.

26. L. C. Young, Generalized surfaces in the calculus of variations. I, Ann. of Math. (2) 43 (1942) 84-103. MR0006023 (3:249a)

27. L. C. Young, Generalized surfaces in the calculus of variations. II, Ann. of Math. (2) 43 (1942) 530-544. MR0006832(4:49d)

Academy of Mathematics and Systems Science, Chinese Academy of Sciences, Beijing 100190, People's Republic of China

E-mail address: libh@amss.ac.cn

Academy of Mathematics and Systems Science and Hua Loo-Keng Key Laboratory of Mathematics, Chinese Academy of Sciences, Beijing 100190, People's Republic of China E-mail address: thli@math.ac.cn 